\title{
„WOHIN MIT DEN INFORMATIONSEXPERTINNEN?“ ERGEB- NISSE EINER ERHEBUNG ZUR BERUFSEINSTIEGSSITUATION DER ABSOLVENTINNEN DES GRUNDLEHRGANGS „LIBRARY AND INFORMATION STUDIES“
}

\author{
von Melanie Nowak und Clara Schneckenleithner
}

Zusammenfassung: In diesem Artikel werden Auszüge aus den Ergebnissen der Projektarbeit „Wohin mit den InformationsexpertInnen? - Arbeitsmarktperspektiven, Beschäftigungsverhältnisse und Berufszufriedenheit nach dem Grundlehrgang Library and Information Studies in Österreich in den Jahren 2010-2015" präsentiert. Die Arbeit entstand im Rahmen des Grundlehrgangs Library and Information Studies (2015/2016) an der Österreichischen Nationalbibliothek unter Zusammenarbeit von Lukas Huber, Melanie Nowak und Clara Schneckenleithner. Mittels einer Online-Umfrage und Auswertung von Stellenausschreibungen wurden Informationen zur Berufseinstiegssituation der Grundlehrgangsabsolventlnnen erhoben. Die Arbeit enthält erste Überlegungen, wie die berufliche Einstiegssituation für AbsolventInnen verbessert werden könnte und bietet eine Grundlage für weitere Analysen zur Arbeitssituation im BID-Bereich.

Schlüsselwörter: Berufseinstieg; Arbeitsmarktperspektiven; Beschäftigungsverhältnisse; Arbeitsanforderungen; Berufszufriedenheit; AbsolventInnen; Library and Information Studies; Grundlehrgang; Projektarbeit

\section{„WHAT TO DO WITH INFORMATION EXPERTS?“ RESULTS OF A SURVEY ON THE LABOR MARIETT SITUATION CONCERNING GRADUATES OF THE BASIC UNIVERSITY COURSE „LIBRARY AND INFORMATION STUDIES“}

Abstract: This article presents extracts from the results of the project work „Wohin mit den InformationsexpertInnen? - Arbeitsmarktperspektiven, Beschäftigungsverhältnisse und Berufszufriedenheit nach dem Grundlehrgang Library and Information Studies in Österreich in den Jahren 2010-2015". The report was written as part of the basic course Library and Information Studies (2015/2016) at the Austrian National Library in cooperation between the participators Lukas Huber, Melanie Nowak and Clara Schneckenleithner. By means of an online-survey and evaluation of job advertisements, information regarding the graduates' entry to the employment market had been collected. The work contains thoughts regarding possible improvements and provides a basis for further analyses of the employment situation in the library and information sector. 
Keywords: career entry; employment market outlook; employment relationship; work requirements; job satisfaction; graduates; Library and Information Studies; basic course; project work

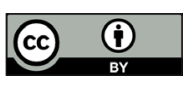

Dieses Werk ist lizenziert unter einer

Creative-Commons-Lizenz Namensnennung 4.0 International

\section{Inhalt}

1. Das Projekt

2. Methoden

3. Ergebnisse Stellenausschreibungen

3.1. Ort und Art der ausschreibenden Einrichtungen

3.2. Aspekte des Arbeitsverhältnisses

3.3. Tätigkeiten und Anforderungen

4. Ergebnisse Online-Umfrage

4.1. Derzeitige Beschäftigung

4.2. Bewerbungsprozess

4.3. Arbeitssuchende

4.4. Anstellungsverhältnisse

4.5. Berufszufriedenheit

4.6. Lehrgangsinhalte

5. Conclusio

\section{Das Projekt}

Während des zehnjährigen Bestehens des Universitätslehrganges Library and Information Studies kam es zu vielen neuen Entwicklungen und Trends im Bibliotheks-, Informations- und Dokumentationswesen. Diese Veränderungen brachten neue Anforderungen für die Bibliotheksorganisation, das Bibliothekspersonal und die Ausbildung mit sich. Vor diesem Hintergrund wurden im Rahmen der Projektarbeit „Wohin mit den Informationsexpertinnen?" die Themen Arbeitsmarktperspektiven, Berufseinstiegssituation und Anforderungen an die AbsolventInnen analysiert.

Der dabei untersuchte Zeitrahmen waren die Jahre 2010 bis einschließlich 2015. Er umfasst alle in diesem Zeitraum liegenden Grundlehrgangsjahrgänge an allen Ausbildungsstandorten Österreichs sowie eine FünfJahres-Analyse (2011-2015) von Stellenausschreibungen.

Da es in Österreich bisher noch keine Erhebung zum Verbleib der AbsolventInnen gab, sollte diese Projektarbeit erste Informationen zum The- 
ma bereitstellen. Derzeit beschäftigt sich die VÖB-Arbeitsgruppe „Prekäre Arbeitsverhältnisse im Bibliothekswesen" eingehend mit dieser Thematik. In Deutschland hat insbesondere die Humboldt-Universität zu Berlin umfangreiche Analysen zum Verbleib der AbsolventInnen in den verschiedenen bibliothekarischen Ausbildungsbereichen vorgenommen. Hier zu nennen seien beispielsweise die Arbeit von Alexandra Puppe „Die Integration der Absolventen des postgradualen Fernstudiums Bibliothekswissenschaft an der HU Berlin in den Arbeitsmarkt: Ergebnisse einer Umfrage zum beruflichen Verbleib“ (2006) sowie eine Verbleibstudie zum Direktstudiengang des Instituts für Bibliotheks- und Informationswissenschaft mit dem Titel „Where did all the good people go?" (2011), die im Rahmen eines Projektseminars erstellt wurde. Diese Studien wurden unter anderem als Input für die Entwicklung dieser ersten Verbleibstudie für Österreich genutzt.

Folgende Forschungsfragen standen am Beginn der Projektarbeit:

- Welche Arbeitsmarktperspektiven gibt es für Absolventlnnen beim Berufseinstieg in den BID-Bereich?

- Mit welchen Beschäftigungsverhältnissen haben Absolventlnnen beim Berufseinstieg in den BID-Bereich zu tun?

- Wie zufrieden sind die im BID-Bereich tätigen AbsolventInnen mit ihrer Arbeitssituation?

- Welche Kompetenzen und Fähigkeiten spielen eine zentrale Rolle beim Berufseinstieg in den BID-Bereich und folglich beim beruflichen Werdegang? Werden diese im Grundlehrgang vermittelt?

- Welche Rolle spielt das Netzwerk des Grundlehrgangs beim Berufseinstieg in den BID-Bereich?

Übergeordnetes Ziel der Projektarbeit war es, eine erste Analyse der aktuellen Arbeitsmarktsituation der AbsolventInnen des Universitätslehrgangs zu bieten, um anschließend als Basis für weitere Erhebungen zu dienen und schließlich zur Verbesserung der derzeitigen Arbeitssituation im BIDBereich beizutragen.

\section{Methoden}

Das Ausgangsmaterial für die Fünf-Jahres-Analyse der Stellenausschreibungen bildeten die vom 1.1.2011 bis zum 31.12.2015 insgesamt 479 Ausschreibungen die über den Blog der Vereinigung Österreichischer Bibliothekarinnen und Bibliothekare veröffentlicht wurden. Bei der Ergebnisauswertung wurden Ausschreibungen für Lehrstellen, Praktika und ähn- 
liches sowie Ausschreibungen für Stellen, die ausschließlich Tätigkeiten außerhalb des BID-Bereichs umfassen, nicht berücksichtigt. Insgesamt wurden somit die Inhalte von 433 Ausschreibungen ausgewertet.

Die Online-Umfrage wurde mittels dem Umfragetool Google Docs erstellt und über die jeweiligen Lehrgangsleiterlnnen an die Grundlehrgangsteilnehmerlnnen (292 versandte Emails davon 4 Fehlermeldungen) der letzten fünf Jahre versandt. Um eine anonyme Beteiligung gewährleisten zu können wurde dabei keine Registrierung (z.B. mittels Emailadresse) eingesetzt, daher kann das theoretische Mehrfachausfüllen des Fragebogens nicht ausgeschlossen werden. Der Fragebogen beinhaltete überwiegend geschlossene Antwortformate. Die Umfrage wurde im Zeitraum vom 9. Juni 2016 bis 15. Juli 2016 durchgeführt und erreichte eine Rücklaufquote von $49,7 \%$.

\section{Ergebnisse Stellenausschreibungen}

\subsection{Ort und Art der ausschreibenden Einrichtungen}

Bei der Auswertung der Stellenausschreibungen wurde zunächst der Ort der über den VÖB-Blog veröffentlichten Stellen erhoben. Von den ausgewerteten Stellen befanden sich rund $89,8 \%{ }^{1}$ in Österreich und rund 10,2\% im Ausland.

Die im Ausland befindlichen Stellen entfielen vor allem auf Deutschland $(31,8 \%)$, die Niederlande $(20,5 \%)$, die Schweiz $(18,2 \%)$ und Italien $(9,1 \%)$.

Die Frage nach der Aufteilung der inländischen Stellenausschreibungen auf die österreichischen Bundesländer brachte folgendes Ergebnis: $74,3 \%$, also fast dreiviertel, entfielen auf Wien, dahinter lagen die Steiermark (5,9\%), Salzburg (5,7\%) und Tirol (4,6\%). Auf die Bundesländer Niederösterreich (2,6\%), Oberösterreich (2,6\%), Kärnten (2,3\%), VorarIberg (1,5\%) und Burgenland (0,5\%) entfielen jeweils zehn oder weniger der 433 ausgewerteten Ausschreibungen.

Bei der Aufteilung der Stellenausschreibungen auf die Bundesländer dominierte Wien also mit bedeutendem Abstand, während in allen übrigen Bundesländern gemeinsam in einem Zeitraum von fünf Jahren insgesamt 100 Ausschreibungen über den VÖB-Blog veröffentlicht wurden.

Ebenso wurde die Aufteilung der Ausschreibungen auf die verschiedenen Arten von Einrichtungen erhoben. Mit 83,4\% beinhaltete der Großteil der ausgewerteten Ausschreibungen eine Stelle in einer bibliothekarischen Ein- 
richtung. 1,2\% der Ausschreibungen entfielen auf Mediatheken, 1,4\% auf Archive, 4,4\% aufForschungseinrichtungen, 6,7\% auf Verbände, Verbünde oder Kooperationen wie zum Beispiel den Österreichischen Bibliothekenverbund (OBVSG), 0,5\% auf Verlage und 2,5\% auf sonstige Einrichtungen. Diese Aufteilung der Ausschreibungen zeigt zwar, dass Bibliothekarlnnen eine breite Palette von Institutionen als mögliche Arbeitsstellen zur Verfügung steht, dass aber im Zeitraum der Erhebung bibliothekarische Einrichtungen als Arbeitsplatz außer Konkurrenz zu anderen Institutionen standen. Da es sich bei den ausgewerteten Ausschreibungen ausschließlich um jene aus dem VÖB-Blog handelt, ist es möglich, dass es zu diesem Ergebnis kommt, weil in erster Linie Bibliotheken den VÖB-Blog als Kommunikationsplattform und Netzwerk nutzen. Anderen Einrichtungen ist dieser Informationskanal möglicherweise nicht bekannt oder er ist für ihre Zwecke nicht attraktiv. Es ist vorstellbar, dass AbsolventInnen freie Stellen und mögliche Arbeitsplätze in Einrichtungen außerhalb der Kategorie „Bibliothek" aufgrund fehlender Informationskanäle nicht berücksichtigen.

\subsection{Aspekte des Arbeitsverhältnisses}

Ebenso wurden Informationen zur Dauer des Arbeitsvertrags erhoben. Von den ausgewerteten Stellenausschreibungen boten 41,1\% die Aussicht auf ein unbefristetes Dienstverhältnis, $51,5 \%$ brachten ein befristetes Dienstverhältnis mit sich, bei 7,4\% der Ausschreibungen konnten keine Angaben ermittelt werden.

Die Stellen mit befristetem Anstellungsverhältnis teilen sich wie folgt auf: Für 0,7\% der ausgewerteten Ausschreibungen war eine Befristung von bis zu drei Monaten vorgesehen, für 5,5\% eine Befristung von bis zu sechs Monaten, für $11,1 \%$ eine Befristung von bis zu einem Jahr, für $16,2 \%$ eine Befristung von bis zu 2 Jahren, für 6,2\% eine Befristung von bis zu 3 Jahren und für 6,0\% war eine Befristung vorgesehen, deren Zeitraum bis zu 4 Jahren oder mehr beträgt (siehe Abbildung 1).

Von den Ausschreibungen für befristete Stellen beinhalteten 2,7\% einen Hinweis auf eine mögliche spätere Verlängerung des Dienstverhältnisses und $8,1 \%$ einen Hinweis auf einen möglichen späteren Wechsel in ein unbefristetes Dienstverhältnis.

Aus der Auswertung der Ausschreibungen geht also hervor, dass ein großer Anteil der ausgeschriebenen Stellen, zumindest zunächst, befristet ist. Wenn man jenen Anteil an Ausschreibungen außer Acht lässt, in welchem keine Angaben zu einer etwaigen Befristung zu finden waren, beträgt die Menge der befristeten Stellen mehr als die Hälfte, nämlich genau 


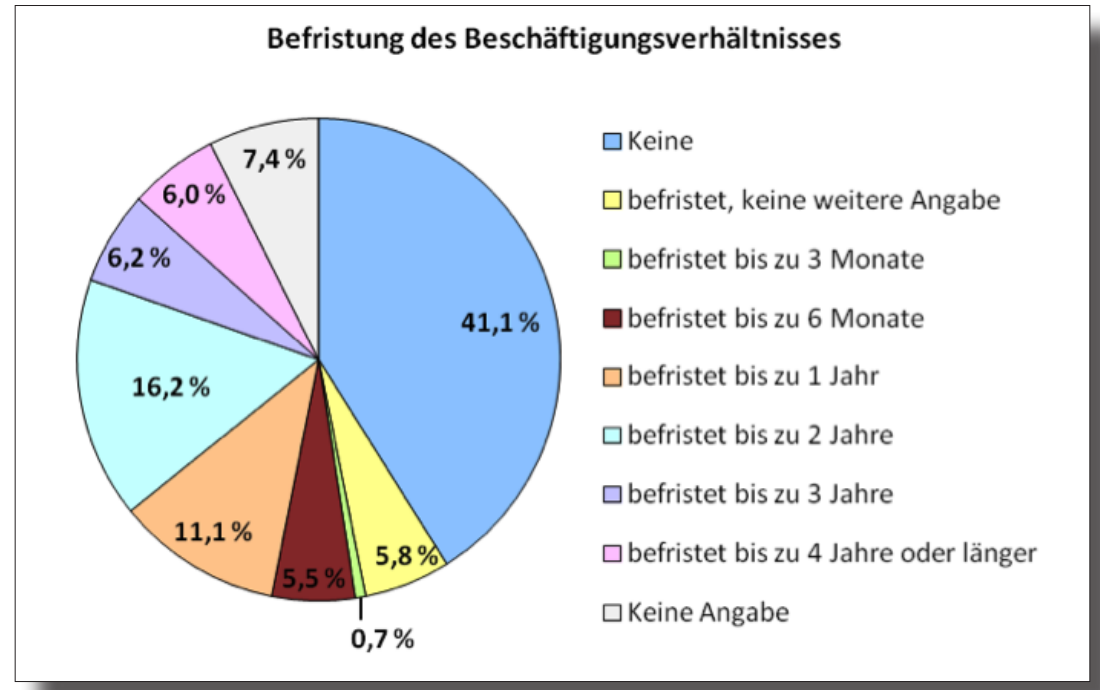

Abb. 1: Prozentuelle Darstellung der Befristungen der Beschäftigungsverhältnisse von 433 Stellenausschreibungen

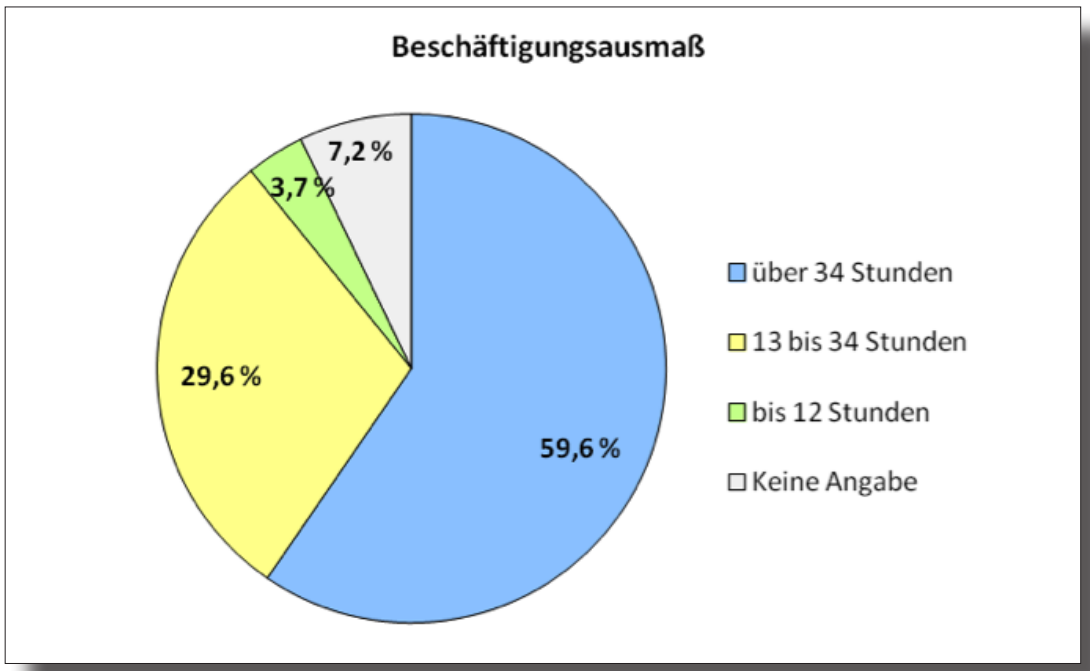

Abb. 2: Prozentuelle Darstellung der Beschäftigungsausmaße von 433 Stellenausschreibungen 
$55,6 \%$. Bei Betrachtung der Stellenausschreibungen, die über den VÖBBlog veröffentlicht werden, gelangt man zu der Annahme, dass die Wahrscheinlichkeit zunächst mit einer befristeten Anstellung in den BID-Bereich einzusteigen in den letzten fünf Jahren höher war als jene mit einem unbefristeten Dienstvertrag einzusteigen.

Ein ähnliches Bild bot sich bei der Erhebung der Wochenstunden der Beschäftigung. 59,6\% der ausgeschriebenen Stellen waren mit einem wöchentlichen Stundenausmaß von mehr als 34 Stunden angegeben. Bei $29,6 \%$ betrug das wöchentliche Stundenausmaß zwischen 13 und 34 Stunden, bei 3,7\% der Stellen betrug es bis zu zwölf Stunden. In 7,2\% der Ausschreibungen konnte kein wöchentliches Stundenausmaß erhoben werden (siehe Abbildung 2).

Lässt man bei der Betrachtung der Auswertung der Kategorie Wochenstunden diejenigen Ausschreibungen außer Acht, in denen keine Angaben zu Wochenstundenanzahl gegeben wurden, beträgt der Anteil an Stellen mit einem Stundenausmaß von mindestens 35 Stunden rund $64,3 \%$. Wenn die Menge der über den VÖB-Blog ausgesendeten Stellenausschreibungen als repräsentativ angenommen wird, kann also die Aussage getroffen werden, dass es im BID-Bereich in den vergangenen fünf Jahren mehrheitlich Ausschreibungen für Stellen mit einem hohen Beschäftigungsausmaß gab.

Die Erhebung von Information zur vorgesehenen Bezahlung der ausgeschriebenen Tätigkeiten gestaltete sich schwierig, da in 43,2\% der Ausschreibungen keine konkreten Angaben zur Entlohnung zu finden waren. Für 3,9\% der Stellen lag das ungefähre monatliche Brutto-Einstiegsgehalt entsprechend einer Vollbeschäftigung bei EUR 1.500.- oder weniger. Bei 31,2\% der Stellen betrug es zwischen EUR 1.501.- und EUR 2.000.und bei 12,9\% der Stellen zwischen EUR 2.001.- und EUR 2.500.-. Bei $5,3 \%$ der Stellen betrug es zwischen EUR 2.501.- und EUR 3.000.-, bei 1,6\% zwischen EUR 3.001.- und EUR 3.500.-, bei 0,7\% zwischen EUR 3.501.- und EUR 4.000.- und bei 1,2\% der Stellen lag es bei über EUR 4.000.-. Um realitätsnahe Aussagen über das durchschnittliche Einstiegsgehalt im BID-Bereich oder auch über Zusammenhänge zwischen Bezahlung und Tätigkeitsbereichen treffen zu können, sind weitere Erhebungen zu dieser Thematik notwendig.

\subsection{Tätigkeiten und Anforderungen}

Die Stellenausschreibungen wurden auch hinsichtlich der angeführten Tätigkeiten ausgewertet. Es wurde in 36,7\% der Stellenausschreibungen eine Beschäftigung im Bereich „Informationsvermittlung und Benutzerlnnenbe- 
treuung" als die hauptsächliche oder eine der hauptsächlichen Tätigkeiten angegeben. Eine Beschäftigung im Bereich „Bestandserschließung“ bzw. "Metadatenmanagement" wurde in 34,0\% der Ausschreibungen angegeben. Als weitere häufig genannte hauptsächliche Tätigkeiten wurden „Bestandsaufbau“ (15,9\%), „systembibliothekarische Tätigkeit“ (12,9\%) und „Management", im Sinne einer Führungsposition, (9,5\%) genannt. Weitere Tätigkeiten wie beispielsweise im IT-Bereich oder im Bereich „Digitalisierung" wurden in 25 oder weniger der 433 ausgewerteten Ausschreibungen als eine der hauptsächlichen Tätigkeiten angegeben (siehe Abbildung 3). Aus 16,2\% der Ausschreibungen ging eindeutig hervor, dass die Anstellung ausschließlich dem Zweck der Mitarbeit an einem Projekt dienen sollte, d.h. die Tätigkeit ausschließlich projektbezogen war.

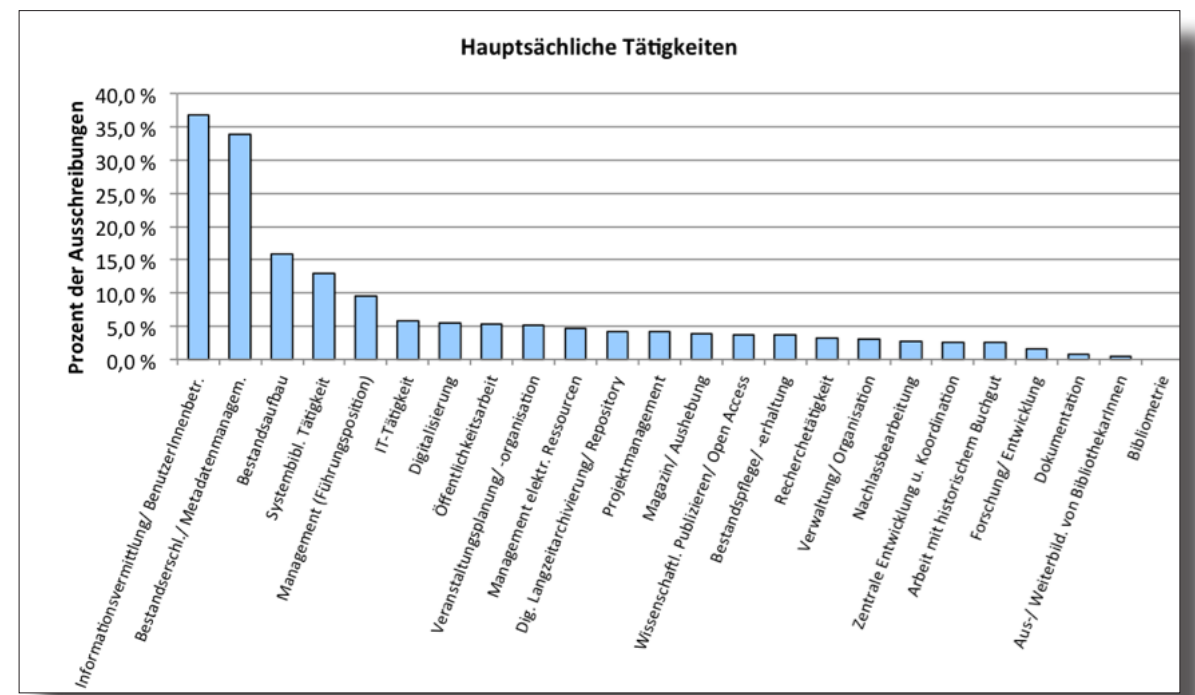

Abb. 3: Prozentuelle Reihung der hauptsächlichen Tätigkeiten, die in 433 Stellenausschreibungen genannt wurden

Auffallend an dem Ergebnis ist das große Spektrum an genannten Tätigkeiten. Dieses wirft die Frage auf, ob es überhaupt möglich ist, das dafür erforderliche breit gefächertes Spektrum an Wissen und Fertigkeiten innerhalb nur eines Lehrgangsjahres zu vermitteln und die AbsolventInnen des Grundlehrgangs auf alle Tätigkeitsbereiche gleichermaßen gut vorzubereiten.

Als die drei größten Tätigkeitsbereiche gehen aus der Auswertung „Informationsvermittlung und Benutzerlnnenbetreuung“, „Bestand- 
serschließung und Metadatenmanagement“, „Bestandsaufbau“ sowie „IT-nahe Tätigkeiten“ hervor, wenn man Systembibliothekarische Tätigkeit, IT-Tätigkeit, Digitalisierung, Management elektronischer Ressourcen und Repository-Management unter letzterem Punkt zusammenfasst.

Die Auswertung der Ausschreibungen brachte ebenso vielfältige Anforderungen seitens der Arbeitgeber hervor. Zu den in den ausgewerteten Stellenausschreibungen am häufigsten geforderten Qualifikationen, Kompetenzen oder Eigenschaften gehörten ein hohes Maß an sozialer Kompetenz $(56,1 \%)$, gute oder sehr gute EDV-Kenntnisse (54,0\%), (mehrjährige) Erfahrung im jeweiligen Tätigkeitsbereich (52,4\%), eine Ausbildung im BIDBereich (46,7\%) und (sehr gute) Englischkenntnisse (45,5\%). Ebenfalls in großem Ausmaß traten die Forderungen nach einem abgeschlossen Studium (31,2\%), nach Anwenderkenntnissen für Bibliothekssoftware (28,6\%) und nach Dienstleistungs-/Service- bzw. Kundenorientierung $(27,7 \%)$ auf. In den beiden folgenden Darstellungen sind weitere Anforderungen angeführt, deren Prozentsatz bei der Erwähnung in den ausgewerteten Ausschreibungen bei ungefähren $20 \%$ oder weniger lag (siehe Abbildung 4 und 5). Nicht in die Grafik aufgenommen wurden jene Anforderungen deren Nennung in weniger als 25 der 433 ausgewerteten Ausschreibungen erfolgte.

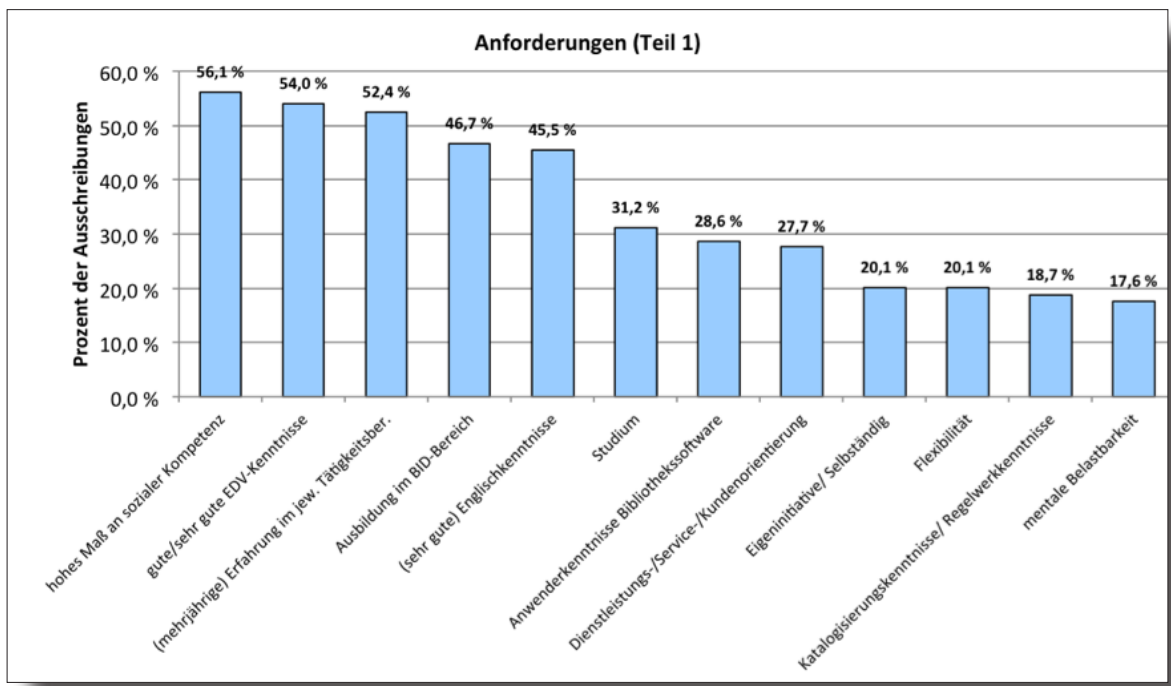

Abb. 4: Prozentuelle Reihung der Anforderungen, die in 433 Stellenausschreibungen genannt wurden 


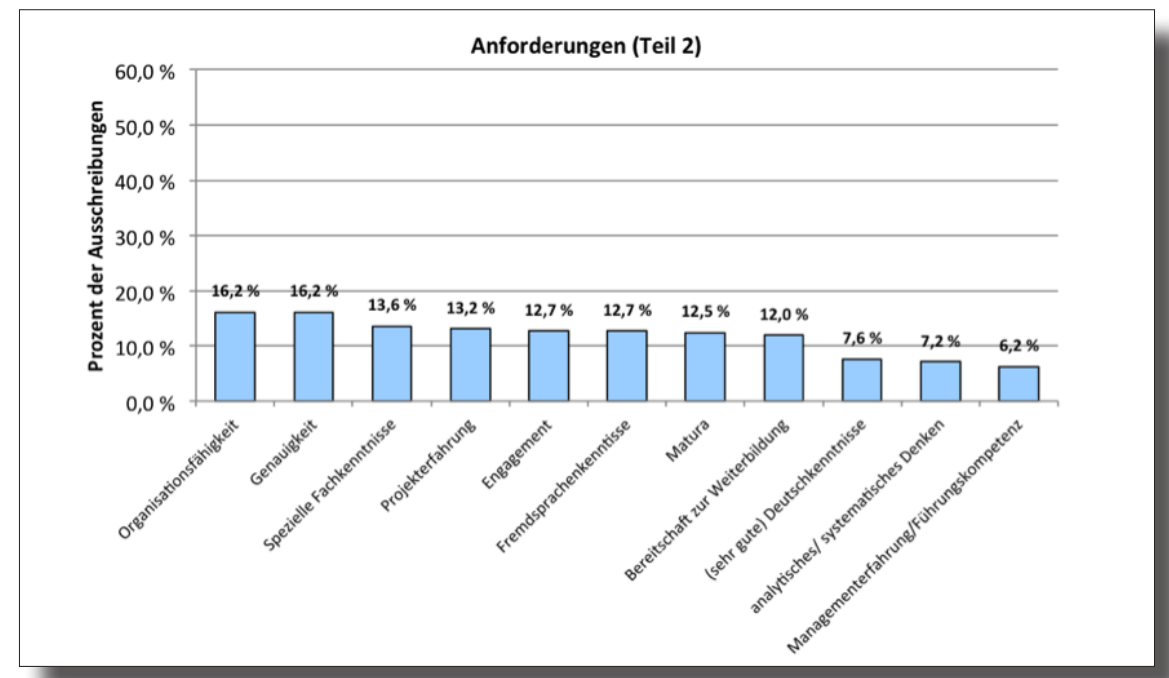

Abb. 5: Prozentuelle Reihung der Anforderungen (Fortsetzung), die in 433 Stellenausschreibungen genannt wurden

Von den 46,7\% der Ausschreibungen, in denen eine Ausbildung im BIDWesen gefordert wurde, untergliederte sich diese Anforderung wie folgt: In 5,5\% der Fälle war eine abgeschlossene Lehre als Archiv-, Bibliotheks- und Informationsassistent/in gefragt. Eine bibliothekarische Ausbildung ohne nähere Angabe, um welche Art von Ausbildung es sich handeln soll, wurde in $63,9 \%$ der Fälle gefordert. Eine bibliothekarische Ausbildung für den wissenschaftlichen bzw. höheren Bibliotheksdienst wurde in 11,9\% der Fälle erwartet. In einigen Ausschreibungen gab es eine explizite Forderung nach dem „Library and Information Studies Grundlehrgang“ (9,4\%) bzw. "Library and Information Studies MSc" (1,0\%).

Bezüglich der Ergebnisse der Auswertung der Anforderungen ist eindeutig, dass eine hohe Nachfrage nach EDV-Kenntnissen gegeben ist. In 54,0\% der Ausschreibungen wurden EDV-Kenntnisse gefordert, 31,2\% davon waren wiederum Forderungen nach sehr guten, speziellen Kenntnissen wie beispielsweise Kenntnis bestimmter Programmiersprachen. Diese Ergebnisse betonen die Notwendigkeit der Vermittlung umfassender EDVbzw. IT-Kenntnisse im Rahmen der bibliothekarischen Ausbildung.

Des Weiteren auffallend war die häufige Forderung nach Anwenderkenntnissen von Bibliothekssystemen, wobei davon in 85,5\% Kenntnisse des Systems ALEPH 500 gefragt waren. Daraus ist abzuleiten, dass es sinnvoll ist im Rahmen des Grundlehrgangs eine intensive Auseinandersetzung 
mit dem jeweiligen im Österreichischen Bibliothekenverbund aktuellen Bibliothekssystem anzubieten.

Aus der Auswertung der Kategorie „Anforderungen“ ist außerdem hervorgegangen, dass die Absolvierung des „Library and Information Studies MSc" kaum (nur in zwei der 433 Ausschreibungen) als Anstellungsvoraussetzung oder Anforderung angegeben wurde. Es ist natürlich nicht möglich Aussagen darüber zu treffen, ob die Absolvierung des Masterlehrgangs trotzdem zu besseren Chancen am Arbeitsmarkt führt. Es lässt sich aber sagen, dass dessen Absolvierung keine Voraussetzung für einen Berufseinstieg ist.

\section{Ergebnisse Online-Umfrage}

\subsection{Derzeitige Beschäftigung}

Die Auswertung des Online-Fragebogens ergab, dass 77,9\% von der Gesamtheit der Teilnehmerlnnen der Umfrage zum Zeitpunkt der Erhebung im bibliothekarischen Bereich tätig waren. Genau 11\% waren auf der Suche nach einer Anstellung im BID-Bereich, ein Teil davon war zu dieser Zeit in einem anderen Arbeitsbereich tätig, der andere Teil war zum Zeitpunkt der Umfrage arbeitslos. Interessant sind die 7,6\% der Befragten die zum Zeitpunkt der Umfrage in einem anderen Arbeitsbereich tätig waren und kein Interesse an einer Anstellung im BID-Bereich hatten. Hier wären weitere Informationen nach den Gründen für die Abkehr aus dem BID-Bereich interessant, nachdem der Grundlehrgang maximal fünf Jahre zurücklag und oft mit Kosten bis zu EUR 5.350,- verbunden ist. Die restlichen 3,4\% der Befragten wurden unter Sonstiges zusammengefasst. Diese befanden sich hauptsächlich in weiteren Ausbildungsschienen (siehe Abbildung 6).

Das Ergebnis zum Zeitpunkt des Berufseinstiegs der AbsolventInnen zeigt, dass 30,3\% der Befragten vor Absolvierung des Grundlehrgangs beruflich in den BID-Bereich eingestiegen sind. Diese Personen sind im Wesentlichen nach Beendigung des Grundlehrgangs wieder zu ihrer früheren Anstellung zurückgekehrt. 11\% der Befragten haben während der Absolvierung des Grundlehrgangs eine Anstellung im BID-Bereich erhalten. Die Mehrheit der Befragten (42,8\%) hat nach Absolvierung des Grundlehrganges eine Anstellung gefunden. 10,3\% der befragten Absolventlnnen der letzten fünf Jahre gaben bezüglich des Zeitpunkts des Berufseinstiegs an auf der Suche nach Anstellung im BID-Bereich zu sein. Zum Zeitpunkt der Umfrage waren 5,5\% der Befragten nicht (mehr) an einer Anstellung im 


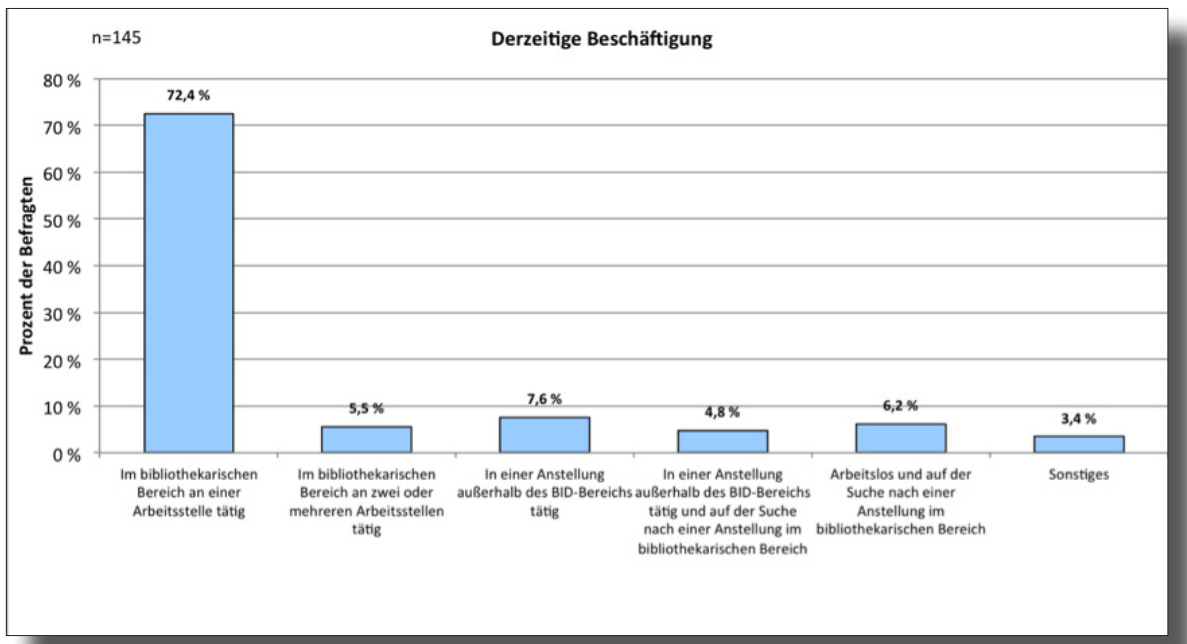

Abb. 6: Verteilung der derzeitigen Beschäftigungssituation der 145 Befragten

\section{Zeitpunkt des Berufeinstiegs in den BID-Bereich}

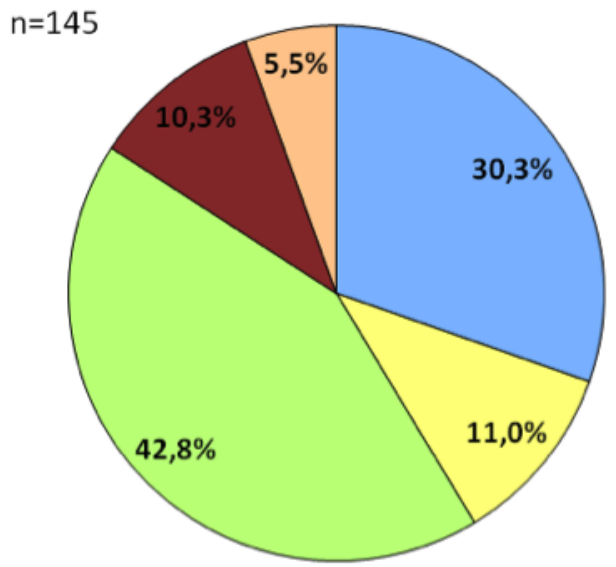

$\square$ Vor Absolvierung des Grundlehrgangs

$\square$ Während der Absolvierung des Grundlehrgangs

Nach Absolvierung des Grundlehrgangs

Ich suche derzeit eine Anstellung im BID-Bereich

$\square$ Ich bin derzeit an einer Anstellung im BID-Bereich nicht interessiert

Abb. 7: Prozentuelle Darstellung des Zeitpunkts des Berufseinstiegs in den BID-Bereich der 145 Befragten 
BID-Bereich interessiert. Mehrheitlich deshalb, weil diese Personen in einer Anstellung in einem anderen Bereich tätig waren (siehe Abbildung 7).

Von den 145 TeilnehmerInnen der Online-Umfrage waren 113 Personen an einer oder mehreren Arbeitsstellen im bibliothekarischen Bereich tätig. Die überwiegende Mehrheit der Befragten (81\% der Nennungen) arbeitete in einer wissenschaftlichen Bibliothek. Auf den Arbeitsbereich der öffentlichen Büchereien entfielen 13,8\% der Nennungen. Unter „Sonstiges“ wurden 5,2\% der Nennungen zusammengefasst. Diese Personen arbeiteten in Arbeitsstellen im BID-Bereich abseits der klassischen Bibliothek wie zum Beispiel in Dachverbänden oder Forschungsservices. In Einrichtungen des Dokumentations- oder Informationswesens waren zum Zeitpunkt der Umfrage kaum AbsolventInnen tätig. Hier stellt sich die Frage, ob es in diesen Bereichen keine Arbeitsstellen gibt, ob die AbsolventInnen Arbeitsstellen in Bibliotheken bevorzugen oder ob die Ausbildung für Bereiche im Informations- oder Dokumentationswesen nicht ausreichend ist. Möglich ist auch, dass es an geeigneten Informationskanälen fehlt und die AbsolventInnen keine Kenntnis von freien Stellen im Dokumentations- und Informationswesen erlangen.

Von den 113 an einer oder mehreren Arbeitsstellen im bibliothekarischen Bereich tätigen Personen, hatten zum Zeitpunkt der Umfrage $73,5 \%$ der Befragten eine Position ohne Führungsverantwortung und $26,5 \%$ der Befragten eine Position mit Führungsverantwortung inne. Dies kann so interpretiert werden, dass allein die Absolvierung des Grundlehrgangs in den darauffolgenden Jahren mehrheitlich nicht zu einer Führungsverantwortung führt.

\subsection{Bewerbungsprozess}

Personen, die während oder nach Absolvierung des Grundlehrgangs in den BID-Bereich eingestiegen sind, wurden zur Thematik des Bewerbungsprozesses befragt. Konkret ging es hierbei um die Bewerbungsanzahl, die Dauer bis zur ersten Anstellung, die Bewerbungsart und Hilfestellungen, die für die Bewerbungen herangezogen wurden.

Bis zur ersten Anstellung im BID-Bereich hatten 20,5\% der Befragten lediglich eine Bewerbung verfasst. Zwischen zwei und fünf Bewerbungen richteten 34,5\% der Befragten bis zur erfolgreichen Anstellung an Institutionen im BID-Bereich. Weitere 32,1\% der Befragten bewarben sich sechs bis 15 Mal. 11,5\% der Befragten verschickten 16 bis 30 Bewerbungen und $1,3 \%$ der Befragten verfassten 31 und mehr Bewerbungen. Dies lässt die Schlussfolgerung zu, dass das Gros der befragten AbsolventInnen bis zur ersten Anstellung zwischen zwei und 15 Bewerbungen verfasste. 
Zur Dauer bis zur ersten Anstellung nach Absolvierung des Grundlehrgangs wurden sowohl Personen, die während oder nach Absolvierung des Grundlehrgangs in den BID-Bereich eingestiegen sind, als auch Personen, die zur Zeit der Umfrage auf der Suche nach einer Anstellung im BID-Bereich waren, befragt.

Hier zeigte sich, dass $28 \%$ der Befragten bereits während der Absolvierung des Grundlehrgangs eine Anstellung im BID-Bereich erhielten. 12\% der Befragten konnten binnen drei Monaten nach Absolvierung des Grundlehrgangs in den BID-Bereich einsteigen. Bis zu sechs Monate nach Absolvierung dauerte es für 19,4\% der Befragten bis sie in einer Anstellung des BID-Bereichs tätig wurden und für 17,2\% der Befragten dauerte es bis zu einem Jahr. 4,3\% der Befragten waren bis zu zwei Jahre nach Absolvierung des Grundlehrgangs auf der Suche nach einer Anstellung im BID-Bereich. Länger als zwei Jahre nach Absolvierung des Grundlehrgangs suchten 1,1\% der Befragten bis sie schließlich eine Anstellung erhielten. Zum Zeitpunkt der Umfrage waren 16,1\% der Befragten auf der Suche nach einer Anstellung im BID-Bereich. Das Ergebnis der Umfrage zeigte, dass 78,6\% der Befragten innerhalb von einem Jahr eine Anstellung im BID-Bereich erhielten.

Im Bezug auf den Bewerbungsprozess zeigte sich zudem, dass sich die überwiegende Mehrheit (80,8\% der Nennungen) der im BID-Bereich tätigen Absolventlnnen für ihre erste Anstellung per Ausschreibung beworben hat. (Praktikums-)Kontakte, Angebote und Blindbewerbungen spielten für den Erhalt der ersten Anstellung im BID-Bereich ebenso eine Rolle wenngleich diese deutlich seltener genannt wurden. Auch Personen die zum Zeitpunkt der Umfrage auf der Suche nach einer Anstellung waren bewarben sich überwiegend über Ausschreibungen (86,7\% der Befragten).

Im Bewerbungsprozess nahmen die AbsolventInnen vor allem den VÖBBlog als Hilfestellung in Anspruch (55,4\% der Nennungen bei den AbsolventInnen für die erste erfolgreiche Anstellung im BID-Bereich und 63,6\% der Nennungen bei den zur Zeit der Umfrage auf der Suche nach einer Anstellung befindlichen Personen). In beiden Gruppen wurden ebenso einschlägige Mailinglisten, Homepages der ausschreibenden Institutionen und das AMS zur Recherche herangezogen. Zusätzlich verhalfen KurskollegInnen, die Lehrgangsleitung, andere Kontakte sowie Jobportale und Internetrecherche einigen AbsolventInnen zu einer erfolgreichen Anstellung im BID-Bereich.

Das Ergebnis zeigt, dass dem VÖB-Blog eine besondere Rolle im Bewerbungsprozess zukommt. Dies könnte ein möglicher Grund dafür sein, dass die meisten AbsolventInnen in einen klassischen bibliothekarischen Bereich einsteigen und kaum jemand in das Dokumentations- oder Informationswesen. 


\subsection{Arbeitssuchende}

Die Umfrage hat ergeben, dass es aus allen befragten Jahrgängen Personen gab, die zum Zeitpunkt der Befragung auf der Suche nach einer Anstellung im BID-Bereich waren. Diese waren entweder in einer Anstellung abseits des BID-Bereichs tätig oder arbeitslos. $50 \%$ der Arbeitssuchenden hatten den letztjährigen Grundlehrgang (2014/2015) als besucht angegeben ${ }^{2}$. Die Arbeitssuchenden wurden auch im Hinblick auf ihre Ausbildungsstätten ausgewertet. Es zeigte sich, dass 62,6\% der Arbeitssuchenden den Grundlehrgang an der Österreichischen Nationalbibliothek absolviert hatten. 25,1\% der Arbeitssuchenden besuchten den Grundlehrgang an der Universitätsbibliothek Wien. Weitere 12,6\% hatten ihre Ausbildung an der Universitätsbibliothek Graz abgeschlossen. Keine Arbeitssuchende gab es in der Umfrage von den Standorten Salzburg oder Innsbruck.

Das Ergebnis der Umfrage zeigte demnach, dass 87,7\% der Arbeitsuchenden an einem der beiden Wiener Standorte ausgebildet wurden. Der Rückschluss es mangle daher an bibliothekarischem Stellenangebot in Wien ist unzulässig, weil die Absolventlnnen ihre Arbeitsstellen nicht zwingend in Wien suchen. Die Wiener Ausbildungsstandorte bilden über ihren eigenen Bedarf aus, um ausgebildetes Bibliothekspersonal für andere Institutionen zur Verfügung zu stellen. Um deutlichere Aussagen treffen zu können, müssten die Arbeitssuchenden nach ihrem angestrebten Arbeitsstandort befragt werden. Zusätzlich wäre eine gesamtösterreichische Erhebung zum Personalbedarf im BID-Bereich interessant. Es bleibt aber der Verdacht, dass in den vergangenen Jahren eine Überausbildung im Standort Wien stattgefunden hat.

\subsection{Anstellungsverhältnisse}

Personen, die zum Zeitpunkt der Umfrage in einer Anstellung im BID-Bereich tätig waren wurden zu ihrem derzeitigen Anstellungsverhältnis befragt. Ebenso wurden Personen, die zum Zeitpunkt der Umfrage in einer Anstellung im BID-Bereich tätig waren und vor Absolvierung des Grundlehrgangs in keiner Anstellung im BID-Bereich tätig waren zu ihren ersten Anstellungsverhältnissen befragt. Konkret wurden die Beschäftigungsausmaße und die etwaigen Befristungen der ersten Anstellung sowie der derzeitigen Anstellung (zum Zeitpunkt der Umfrage) erhoben.

Bezüglich der Beschäftigungsausmaße zeigte sich, dass die Mehrheit der Befragten in den vergangenen Jahren mit einer Stundenzahl wie sie meist eine „geringfügigen Beschäftigung" aufweist, oder mit einer Teilzeitanstellung (58,3\% der Befragten) in den BID-Bereich eingestiegen ist. $41 \%$ 
der Befragten hatten im ersten Anstellungsverhältnis ein Beschäftigungsausmaß von über 34 Stunden (Abbildung 8).

\section{Beschäftigungsausmaß des ersten Anstellungsverhältnisses}

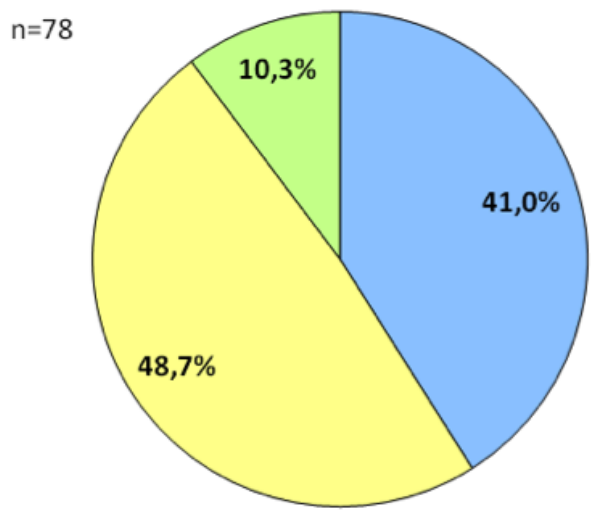

$\square$ über 34 Stunden

$\square 13$ bis 34 Stunden

$\square$ bis 12 Stunden

Abb. 8: Prozentuelle Zusammensetzung des Beschäftigungsausmaßes (in Stunden) des ersten Anstellungsverhältnisses, 78 Betroffene von 145 Befragten

Ein anderes Bild hinsichtlich Beschäftigungsausmaß ergab sich bei den derzeitigen Anstellungen (zum Zeitpunkt der Umfrage). Hier zeigte sich, dass die Mehrheit in einem Anstellungsverhältnis über 34 Stunden (67\% der Nennungen) tätig war. 32,2\% der Nennungen entfielen auf Teilzeitanstellungen im Bereich von 13 bis 34 Stunden, bis zu 12 Stunden waren kaum noch AbsolventInnen (0,9\% der Nennungen) im BID-Bereich beschäftigt (Abbildung 9).

Zur Thematik des Beschäftigungsausmaßes kann zusammenfassend gesagt werden, dass die Beschäftigung im BID-Bereich mehrheitlich mit einer Teilzeitanstellung beginnt. Im derzeitigen Beschäftigungsverhältnis verbessert sich die Situation. Hier muss berücksichtigt werden, dass Personen die möglicherweise keine Verbesserung erzielen konnten, eventuell auch die Branche gewechselt haben. Personen, die bereits im BID-Bereich vor Absolvierung des Grundlehrgangs tätig waren, verbessern das Ergebnis zum derzeitigen Beschäftigungsausmaß insofern, als viele von diesen mit höheren Stundenausmaßen beschäftigt sind. Allerdings zeigt sich auch für die neu eingestiegenen AbsolventInnen, dass sich das Stundenausmaß hauptsächlich verbessert oder gleich bleibt. Selbiges gilt auch für die Befristungen. 


\section{Derzeitiges Beschäftigungsausmaß}

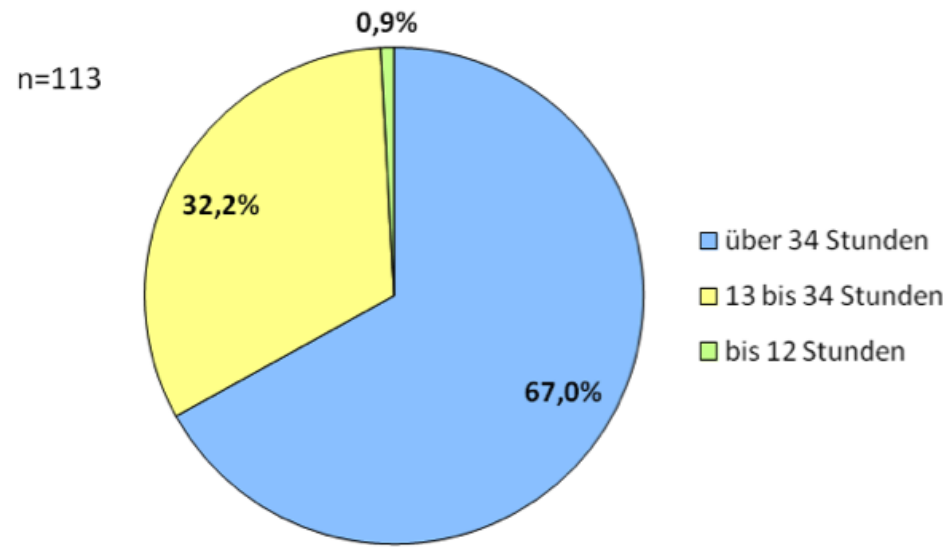

Abb. 9: Prozentuelle Zusammensetzung des derzeitigen Beschäftigungsausmaßes (in Stunden), 113 Betroffene von 145 Befragten

Bei den Befristungen zeigte sich ein ähnliches Bild wie bei den Beschäftigungsausmaßen. Beim ersten Anstellungsverhältnis erhielt der Großteil der Befragten eine befristete Anstellung (78,2\% der Befragten). Für 21,8\% der Befragten war die erste Anstellung unbefristet. Die Mehrheit der befristeten Anstellungsverhältnisse wies eine Befristung zwischen sechs Monaten und einem Jahr auf (Abbildung 10).

Die derzeitigen Beschäftigungsverhältnisse zeigten, ähnlich wie beim Beschäftigungsausmaß, eine deutliche Änderung gegenüber dem ersten Anstellungsverhältnis. 30,8\% der Nennungen waren befristete Anstellungsverhältnisse, mehrheitlich befristet auf bis zu einem Jahr oder zwei Jahre. Der Großteil der Befragten (69,2\% der Nennungen) hatte ein Anstellungsverhältnis ohne Befristung (Abbildung 11).

Bei der Thematik der Befristungen zeigte sich also, dass die Mehrheit mit einem befristeten Vertrag in den BID-Bereich eingestiegen ist. Im derzeitigen Beschäftigungsverhältnis hingegen sind die unbefristeten Anstellungsverhältnisse in der Mehrheit. Auch ohne die Personen, die vor Absolvierung des Grundlehrgangs in den BID-Bereich eingestiegen sind, zeigt sich eine Verbesserung für viele Absolventlnnen in Form von einem unbefristeten Anstellungsverhältnis oder längeren Befristungen. Hier muss erwähnt werden, dass ein befristetes Anstellungsverhältnis per se nicht negativ sein muss. Problematisch werden diese allerdings, wenn keine unbe- 


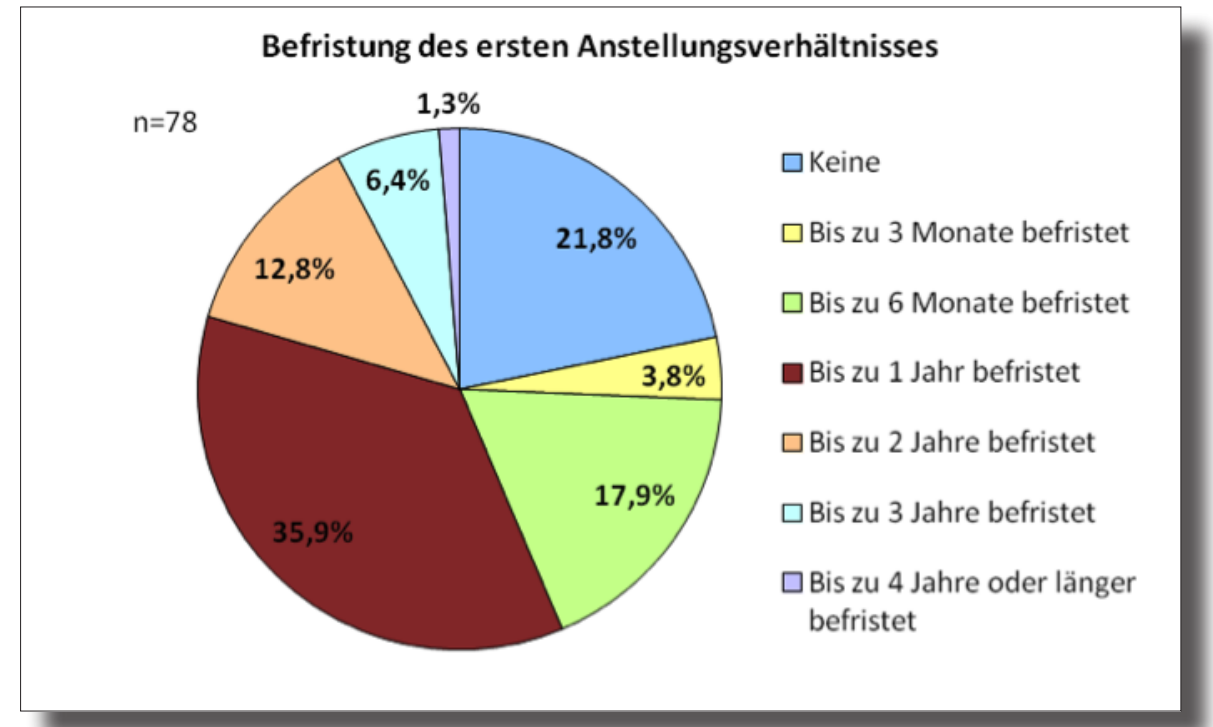

Abb. 10: Prozentuelle Übersicht über die Dauer der Befristung des ersten Anstellungsverhältnisses, 78 Betroffene von 145 Befragten

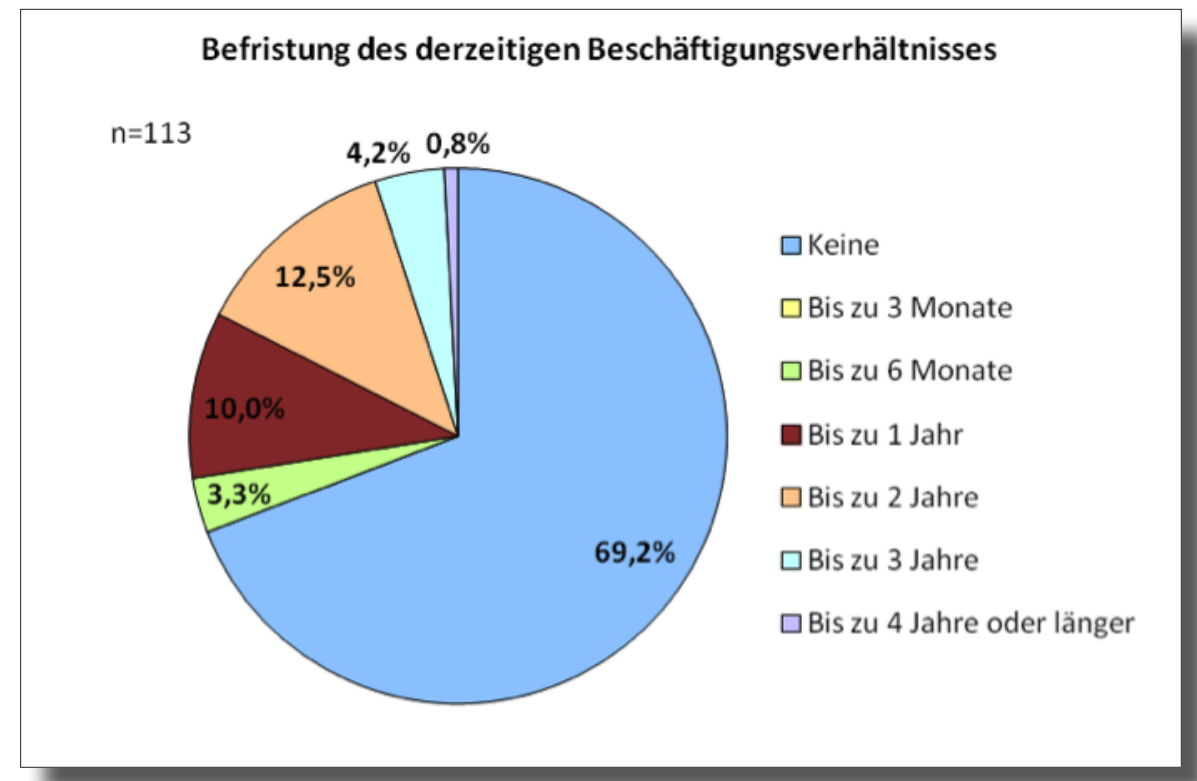

Abb. 11: Prozentuelle Übersicht über die Dauer der Befristung des derzeitigen Anstellungsverhältnisses, 113 Betroffene von 145 Befragten 
fristeten Verträge in Aussicht sind, oder Personen jahrelang ungewollt von kurz befristeten Projektanstellungen betroffen sind.

\subsection{Berufszufriedenheit}

Die Erhebung hat eine hohe Berufszufriedenheit der im BID-Bereich tätigen AbsolventInnen ergeben. 91,6\% der Befragten sind sehr zufrieden oder zufrieden, anteilsmäßig ca. jeweils die Hälfte. Bezahlung (34,8\% der Nennungen) gefolgt von Beschäftigungsausmaß und Befristungen (jeweils 20,9\% der Nennungen) sind die Aspekte, die am öftesten gerne an der Arbeitssituation verändert würden.

Die Absolvierung des Grundlehrgangs hatte für viele Personen (44,9\% der Nennungen), die vor Absolvierung des Grundlehrgangs im BID-Bereich angestellt waren keine Auswirkung auf die Arbeitssituation. Die am Öftesten genannte Änderung in der Arbeitssituation war der Wechsel in eine höher qualifizierte Position (28,6\% der Nennungen). Jeweils 8,2\% der Nennungen entfielen auf das Beziehen eines höheren Gehaltes oder den Wechsel in eine Position mit Führungsverantwortung, alle weiteren Nennungen wurden unter „Sonstiges“ zusammengefasst.

\subsection{Lehrgangsinhalte}

Die Absolventlnnen des Grundlehrgangs, die nach oder während der Absolvierung des Grundlehrgangs in den BID-Bereich eingestiegen sind, gaben eine Vielzahl von Inhalten an, die sie zum Berufseinstieg qualifizierten. Die Antworten auf diese Frage sind sehr heterogen. Deutlich zeigte sich aber, dass in den vergangenen Jahren der Bereich der Formalerschließung (und Sacherschließung) der wichtigste Lehrgangsinhalt war. Für diesen Bereich sollte insbesondere durch die Umstellung auf RDA, ausreichend Zeit im Grundlehrgang veranschlagt werden.

Interessant ist auch, dass ein Teil der Befragten (9,5\% der Nennungen) den Grundlehrgang als guten Überblick und Basis für den Berufseinstieg betrachtete, hingegen ein anderer Teil die bloße Absolvierung, also das Zertifikat, als wichtig für den Berufseinstieg empfand (7,8\% der Nennungen). Auch Informationsrecherche und Informationskompetenz hielten einige Personen (8,6\% der Nennungen) für Inhalte, die zum Berufseinstieg qualifizierten. Für Personen, die in öffentlichen Büchereien eine Anstellung erhielten, waren zweifelsfrei die Wahlmodule „Öffentliche Büchereien I \& II“ für den Berufseinstieg relevant (6\% der Nennungen). Diese optionalen Module sind für den Berufseinstieg eine 
wichtige Zusatzqualifikation. Auch die Praktika (5,2\% der Nennungen) scheinen für den Berufseinstieg oft wichtige Kontaktmöglichkeiten zu sein (siehe Abbildung 12).

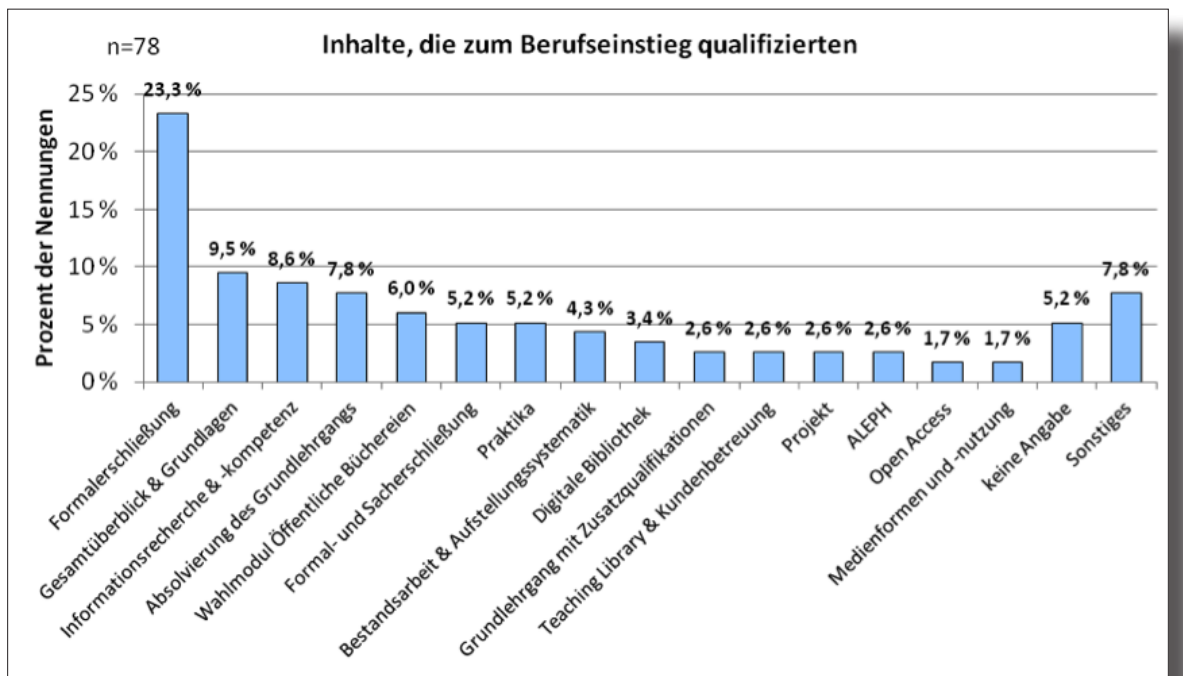

Abb. 12: Reihung der Inhalte, die laut der 78 Befragten zum Berufseinstieg qualifizierten

Auch die Arbeitsgebiete auf die der Grundlehrgang nicht vorbereitet hat sind den Antworten zufolge mannigfaltig. Unter Sonstiges, der höchste Balken, wurden Nennungen zusammengefasst, die allesamt nur einmal genannt wurden (21\% der Nennungen). Die Formalerschließung (9,5\% der Nennungen), wobei hier oft Spezialgebiete der Formalerschließung genannt wurden, wurde auch bei den Arbeitsgebieten auf die der Grundlehrgang nicht vorbereitete am öftesten genannt. Mehrmals wurde betont, dass dies sehr spezielle Arbeitsbereiche sind, die von vielen Personen im Arbeitsalltag nicht gebraucht würden. Fast ebenso vielen Personen (8,4\% der Nennungen) fehlten im Grundlehrgang keine Inhalte. Diese Personen waren mit dem Grundlehrgang sehr zufrieden. Teaching Library \& Kundenbetreuung wurde nach Angabe von sieben Teilnehmerlnnen (6,7\% der Nennungen) unzureichend im Grundlehrgang thematisiert. Auf diese Nachfrage wurde bereits mit dem Curriculum 2013 reagiert - seitdem beinhaltet der Grundlehrgang Lehrveranstaltungen zu den Themen „Teaching Library" und "Serviceorientierung“. Zählt man die Bereiche e-Medien Verwaltung, Systembibliothekarische Tätigkeiten und Datenmanagement zusammen (15,2\% der Nennungen) so zeigt sich, dass die Inhalte der Digitalen 
Bibliothek wichtige berufliche Inhalte sind, die es zu berücksichtigen gilt. Ebenso sind klassische bibliothekarische Tätigkeiten, wie Formalerschließung oder auch Kundenbetreuung, wichtige Inhalte des Berufsfelds und daher ausbildungsrelevant (siehe Abbildung 13).

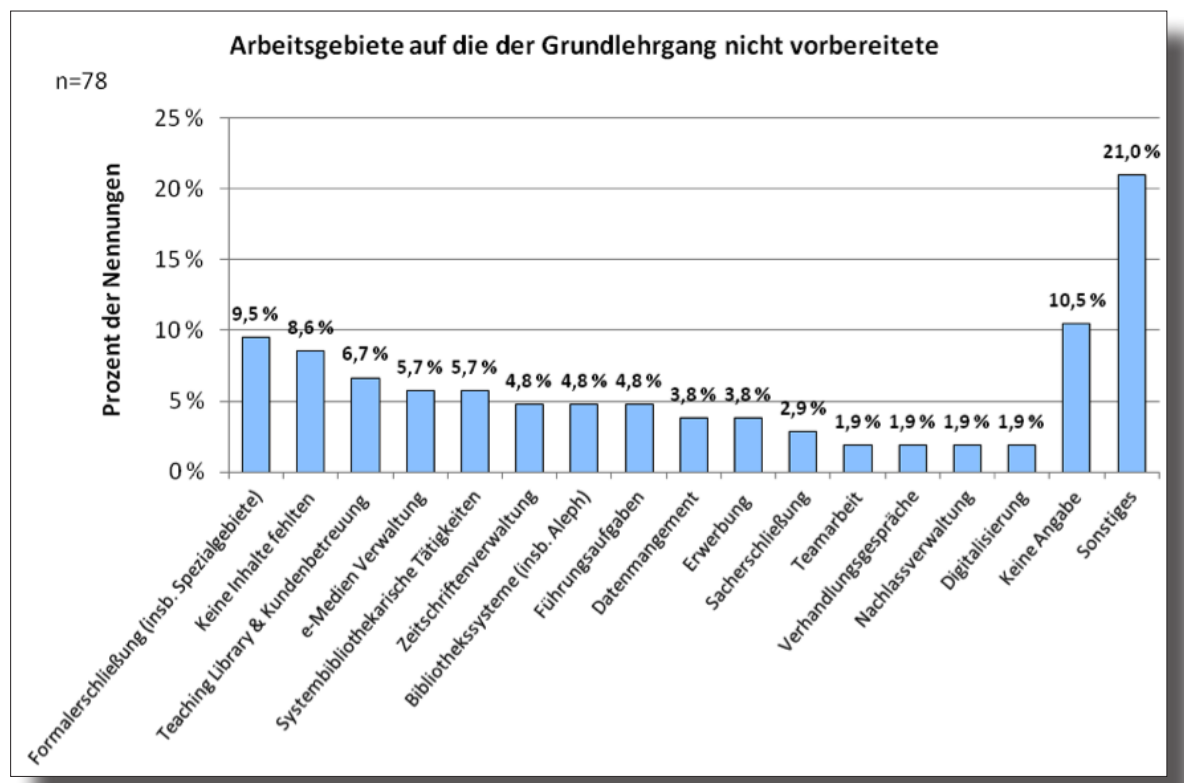

Abb. 13: Reihung der Arbeitsgebiete auf die der Grundlehrgang laut der 78 Befragten nicht vorbereitete

Zur Thematik der Lehrgangsinhalte können also folgende Aussagen getroffen werden: Sowohl bei den Inhalten die qualifizierten als auch bei den Arbeitsgebieten auf die nicht vorbereitet wurde zeigte sich ein überaus heterogenes Antwortspektrum. Dies zeigt wie vielfältig der BID-Bereich ist und stellt den Grundlehrgang vor die Problematik auf die oft sehr verschiedenen Arbeitsgebiete vorbereiten zu müssen. Generell zeigt sich aber auch, dass ein Teil der Befragten durchaus mit der Ausbildung zufrieden war. Wichtig ist, dass die „alten Kernkompetenzen“ des bibliothekarischen Berufs, insbesondere die Formalerschließung und Informationsrecherche und -kompetenz, von den Befragten nach wie vor als wichtige Inhalte betrachtet werden. Ebenso zeigt sich, dass die Inhalte der Digitalen Bibliothek wichtig für die Berufsausbildung sind. 


\section{Conclusio}

Auf Basis der vorliegenden Ergebnisse ergaben sich für uns folgende Schlussfolgerungen:

- Der Grundlehrgang wird von einigen Teilnehmerlnnen der Online-Umfrage als solide Grundausbildung angesehen, welche gut auf den BIDBereich vorbereitet.

- Die Auswertung der Ergebnisse deutet darauf hin, dass vertiefende Praxis-Wahlfächer zu den im Grundlehrgang bereits behandelten Themen wie Informationstechnologie, Repositorien oder Kundenbetreuung aufgrund der hohen Nachfrage auf Arbeitgeberseite sinnvoll wären. Andere Themenbereiche der Wahlfächer, wie beispielsweise Bibliometrie, werden in Stellenausschreibungen praktisch nicht nachgefragt.

- Sowohl in den Stellenausschreibungen als auch bei der Online-Umfrage wurde immer wieder auf die benötigte Kompetenz der Anwendung von Bibliothekssystemen hingewiesen. Es stellt sich die Frage, ob vermehrte Praxiseinheiten für beispielsweise ALEPH beziehungsweise in Zukunft ALMA sinnvoll wären.

- Elf Prozent der in der Online-Umfrage befragten AbsolventInnen waren zum Zeitpunkt der Umfrage auf der Suche nach einer Anstellung im BID-Bereich. Weitere Erhebungen zu den Umständen (erstrebter Anstellungsort, unerfüllte Anforderungen von Seiten der AbsolventInnen, Gründe für Bewerbungsabsagen etc.) in denen sich die arbeitsuchenden AbsolventInnen befinden sind für eine umfassende Beleuchtung der Thematik erforderlich.

- Der BID-Bereich stellt sich als äußerst heterogenes Berufsfeld dar. Diese Heterogenität wurde sowohl anhand der Auswertungen der Stellenausschreibungen (Anforderungen) als auch anhand der Auswertungen der Online-Umfrage (Inhalte und Arbeitsgebiete) sichtbar. Für die Konzeption des Grundlehrgangs bedeutet das, dass auf diese Fülle von Anforderungen reagiert werden muss. Wir schlagen folgende mögliche Zukunftsszenarien für den Grundlehrgang vor, um die Berufseinstiegssituation der AbsolventInnen zu verbessern:

1. Der Grundlehrgang wird in gleicher Form wie bisher beibehalten. Einige TeilnehmerInnen der Online-Umfrage waren schließlich sehr zufrieden. Zwar könnten nicht alle Anforderungen im Grundlehrgang umfassend behandelt werden, der Grundlehrgang könnte aber eine gute Basis sein. Die Kenntnisse würden in weiterer Folge nach dem Berufseinstieg mittels „learning on the job“ erweitert und vertieft werden. Um eine bessere Unterbringung der AbsolventInnen am 
Arbeitsmarkt zu gewährleisten könnte der Grundlehrgang in einer geringeren Frequenz abgehalten werden.

2. Der Grundlehrgang bleibt einjährig dafür spezialisieren sich die Lehrgangsteilnehmerlnnen innerhalb des Lehrgangs. Aufgrund der Fülle der Anforderungen ist eine intensive Beschäftigung mit allen Bereichen des Bibliotheks-, Informations- und Dokumentationswesen nicht mehr möglich. Nach einem allgemeinen Grundmodul könnten sich die Lehrgangsteilnehmerlnnen auf zwei Bereiche spezialisieren z.B. Teaching Library/Kundenbetreuung, Systembibliothekarische Tätigkeiten oder Informationsrecherche/-kompetenz. Problematisch ist dies insofern, als dass die Lehrgangsteilnehmerlnnen im Bewerbungsprozess aufgrund ihrer Spezialisierungen eingeschränkter wären.

3. Der Grundlehrgang wird in seiner Dauer verlängert, um die verschiedensten Anforderungen ausreichend behandeln zu können. Um auf die Situation der Arbeitssuchenden zu reagieren, könnten insbesondere auch Inhalte abseits des klassischen bibliothekarischen Arbeitsgebiets integriert werden, beispielsweise solche, die für Tätigkeiten im Dokumentations- oder Informationswesen befähigen. Hier könnten insbesondere auch Arbeitsbereiche für die Informationsorganisation im digitalen Zeitalter berücksichtigt werden.

- Die Berufszufriedenheit der im BID-Bereich tätigen AbsolventInnen ist sehr gut. Die Bereiche Bezahlung, Beschäftigungsausmaß und Befristungen wurden von den Teilnehmerlnnen der Online-Umfrage als potenzielle Verbesserungsfelder der Arbeitssituation aufgezeigt. Zu diesen Thematiken sind weitere Analysen notwendig.

Neben vielfältigen Ideen zur Berufseinstiegssituation der GrundlehrgangsabsolventInnen haben die Ergebnisse der Projektarbeit auch eine Reihe von weiteren Fragen aufgeworfen, wie zum Beispiel:

- Unterscheiden sich die durchschnittlichen Bruttogehälter im Bezug auf die verschiedenen Tätigkeitsbereiche im BID-Bereich?

- Welche Arbeitsorte sind von AbsolventInnen nachgefragt und wie ist dort der Bedarf?

- Worin liegen die Gründe für die Abwendung von AbsolventInnen vom BID-Bereich?

Die Projektarbeit wurde mit dem Ziel verfasst einen ersten Überblick über die derzeitige Lage zu geben sowie die Ergebnisse als Grundlage für weitere Analysen zur Ausbildung und Arbeitssituation im BID-Bereich zu nutzen. Die Projektarbeit wurde nach einem erst kurzen Einblick in den BID-Bereich 
und aus der Perspektive von Berufseinsteigerlnnen verfasst. Nach Beendigung des Projekts und mit zunehmender Kenntnis über das Bibliotheks-, Informations- und Dokumentationswesen, haben sich für uns weitere Verbesserungspotenziale innerhalb der Arbeit erschlossen.

Melanie Nowak Universitätslehrgang Library and Information Studies 2015/16 Österreichische Nationalbibliothek Josefsplatz 1, A-1015 Wien E-Mail: ulg@onb.ac.at; lis.berufseinstiegsstudie@gmail.com

Clara Schneckenleithner BA BA Universitätslehrgang Library and Information Studies 2015/16 Österreichische Nationalbibliothek Josefsplatz 1, A-1015 Wien E-Mail: ulg@onb.ac.at; lis.berufseinstiegsstudie@gmail.com

\section{Literatur}

Gäde, Maria; Pannier, Gertrud; Petras, Vivien et al. (2011): Where did all the good people go? Online unter: http://www.ib.hu-berlin.de/verbleibstudie/projektbericht.htm [Zugriff: 13.02.2017]

Puppe, Alexandra (2006): Die Integration der Absolventen des postgradualen Fernstudiums Bibliothekswissenschaft an der $\mathrm{HU}$ Berlin in den Arbeitsmarkt. Ergebnisse einer Umfrage zum beruflichen Verbleib, Berlin: Institut für Bibliotheks- und Informationswissenschaft der HumboldtUniversität zu Berlin (Berliner Handreichungen zur Bibliotheks- und Informationswissenschaft 163).

Universität Wien (2013): Curriculum für den interuniversitären Universitätslehrgang „Library and Information Studies" (Grundlehrgang und MSc) an den Universitäten Wien, Graz, Innsbruck und Salzburg (Version 2013). Online unter: http://www.univie.ac.at/ mtbl02/2012_2013/2012_2013_245.pdf [Zugriff: 13.02.2017]

1 Prozentzahlen wurden in der Arbeit auf eine Kommastelle gerundet, daher können die Ergebnisse geringfügig über oder unter $100 \%$ betragen.

2 Eine Person konnte keinem Ausbildungsjahrgang zugeordnet werden, da die Frage nach dem Ausbildungsjahrgang zur Wahrung des Datenschutzes fakultativ war. 